\title{
Migraine in children under 6 years of age: A long-term follow-up study
}

\author{
Francesca Marchese a, ${ }^{\text {, }}$ Luciana Rocchitelli a, ${ }^{\text {, }}$ Luca Maria Messina a , Rosaria Nardello ${ }^{\text {a }}$ \\ Giuseppe Donato Mangano a, Francesca Vanadia ${ }^{\text {b }}$, Salvatore Mangano a, \\ Filippo Brighina ${ }^{\mathrm{c}}$, Vincenzo Raieli ${ }^{\mathrm{b}, *}$ \\ a Child Neuropsychiatry Unit Department Pro.M.I.S.E. “G D'Alessandro”, University of Palermo, Italy \\ b Child Neuropsychiatry Dept., P.O. Di Cristina, ARNAS Civico Palermo, Italy \\ ${ }^{\mathrm{c}}$ Department of Experimental Biomedicine and Clinical Neurosciences, University of Palermo, Italy
}

\section{A R T I C L E I N F O}

\section{Article history:}

Received 15 July 2019

Received in revised form

23 September 2019

Accepted 13 April 2020

\section{Keywords:}

Pediatric headaches

Children

Migraine

Allodynia

Cranial autonomic symptoms

\begin{abstract}
A B S T R A C T
Background: Early starting of migraine seems predictive for less favorable outcome in later ages, however follow-up investigations are very few and all with short-term prospective period. We report here the longest follow-up study in a population of children presenting with migraine under the age of 6.

Methods: We followed-up 74 children under 6 years of age, referred for headache to our department between 1997 and 2003. The study was carried out between October 2016 and March 2018. Headache diagnoses were made according to the IHS criteria.

Results: $23 / 74$ patients, $31 \%$ of the original cohort, were found at follow-up in a period ranging between 15 to 21 years after the first visit. Seven of them were headache free. The remaining 16 patients had migraine. In the migraine group, the localization of pain changed in $75 \%$ of the subjects, $11 / 16(68.7 \%)$ had allodynia and 9/16 (56.25\%) had cranial autonomic symptoms.

Conclusions: Our results suggest that the onset of migraine at very young age represents unfavorable prognostic factor for persistence of the disease at later ages. Some clinical features may change during clinical course, and the active persistence of the disorder may lead to an increase in allodynia.
\end{abstract}

(C) 2020 Published by Elsevier Ltd on behalf of European Paediatric Neurology Society.

\section{Introduction}

Migraine is a common and disabling neurological disorder in the pediatric population, but it has received limited attention in children below 6 years, as only a few studies have so far provided a careful description of the clinical features of and therapeutic options for migraine in this age group [1].

Recent studies highlighted the increasing prevalence of pediatric primary headache/migraine in both outpatients and in pediatric emergency departments [2-14]. Even if based on a few reports, the acquired evidence sufficiently demonstrated the main epidemiological and clinical features of primary headaches, especially migraine, in preschool-age children. Migraine is a life-long, intermittent illness with a serious impact on quality of life that should be considered in the planning of the therapeutic strategy. Concerns over the long duration of migraine seem to be more relevant to children than to their parents or paediatricians [15]. Surely the early onset of the disorder raises important questions about the future of younger children, along with their families, who sense the prospect of possible lasting suffering and disability for their children. In addition, the early onset of migraine is a relevant issue that needs to be investigated in order to understand the relationship with the evolution of the disorder. Unfortunately, only few studies have explored migraine in preschoolers. Prospective follow-up investigations are even rarer, and all have explored a relatively short period, not longer than ten years in any case $[8,9,16,17]$.

We present here the longest follow-up study in a population of children with migraine onset below 6 years of age (when they were studied for first time).

\footnotetext{
* Corresponding author. via dei Benedettini 1, P.O. Di Cristina, 90100, Palermo, Italy. E-mail address: vincenzo.raieli@arnascivico.it (V. Raieli).

1 These authors contributed equally.
} 


\section{Methods}

The study was approved by the local Ethics Committe (statement n. 9/2016), and written consent was obtained from interviewed subjects or from their guardians. The follow-up was carried out between October 2016 and March 2018. The research involved children under the age of 6 who had been referred to Child Neuropsychiatry Department between 1997 and 2003 (T0) for primary headaches [7], and consisted of two steps. The first one was to find patients and to learn the basics about their headaches. This was accomplished through a phone call to the patients or their tutors asking about the disorder outcome (persistence, remission, duration of headache attacks) and their willingness to take part to a further direct interview in our department, which represented the second step of the study. Only subjects with persistent headache attacks were later interviewed in our Center (T1). Unfortunately, we were only able to reach a few patients this way, because most phone numbers in our original records were useless or lost due to the recent widespread use of mobile phones. Accordingly, we looked for the remaining names by searching within the lists of the local public healthcare agency of the municipality of Palermo, where all residents are recorded. These lists report the names of all patients and the addresses (including phone numbers) of the family doctors who take care of them. We contacted the doctors in order to obtain patients' phone numbers. Thanks to these strategies, we found 23 subjects. Despite a lot of motivation and effort we were not able to obtain information about the outcomes for the remaining 51 subjects. There were different causes for this: subjects were not included in the Palermo province patient list, doctors or patients refuses to cooperate, phone numbers were non-existent, or there was no answer to repeated phone calls.

Four subjects refused the phone interview, for them, should be possible to hypothesize that this refusal could depend on the headache disappearance. However, three of them originally had another type of headache and for this reason they would not have anyway be included within the follow-up.

At the end of the follow-up, we updated the clinical course of 23 subjects through direct interviews regarding the cephalalgic attack persistence and the main associated features of their attacks [18].

At T0 the original cephalalgic population was classified using the diagnostic criteria of 1st International Classification Headache Disorders (1988) [19], while at T1 we used the diagnostic criteria of 3rd International Classification of Headache Disorders (2013 beta version) [20]. However, we underline that the diagnostic criteria for migraine without aura remained unchanged.

All data were recorded using Microsoft Excel. All calculations were performed in Excel, and we used $\chi$ [2] to compare the percentages.

\section{Results}

\subsection{Distribution sample at time 0 (T0)}

Between 1997 and 2003, we enrolled our first cohort of 74 subjects, who had a mean age of $4.8 \pm 1.3$ years (range 17-71 months) at first medical consultation. The cohort included 37 children $(22 \mathrm{M} / 15 \mathrm{~F})$ with migraine, 19 with episodic tension headaches (12 M/7 F), 13 with primary stabbing headaches (7 M/ $6 \mathrm{~F})$, and 5 with chronic daily headaches (2 F/3 M).

\subsubsection{Distribution sample at time (T1)}

At time T1, after a follow-up period ranging from 15 to 21 years (mean duration 17.4 years), we interviewed 23/74 (31\%) subjects in the early sample. Seven of them were headache-free, and the remaining 16 were affected by migraine.

Seventeen of the 23 patients found at follow-up were originally migraineurs ( $17 / 37$, or $45.9 \%$ of the total early migraine sample), with 3 others suffering from primary stabbing headaches $(3 / 13,23 \%$ of early sample) and 3 from tension-type headaches (3/19, or $15.7 \%$ of early sample).

At T1, 13/17 (76.47\%) original migraineurs patients still complained migraine attacks ( 5 reported a better headache course, 5 a worse course and 3 the same clinical course); the remaining 4 subjects were attack-free.

Two further subjects with previous primary stabbing headaches and one with episodic tension type headaches changed their headache type to migraine at $\mathrm{T} 1$, while the remaining three subjects were attack-free. In total, we had 16 subjects with migraine attacks at T1. Demographic features (age and sex) of migraine subjects at T0 and $\mathrm{T} 1$ are reported in Table 1.

\subsection{Clinical features of pain sample at $T 1$}

To evaluate the main clinical features of the sample of actual migraineurs and the changes in it from T0 to T1, we also included the 3 subjects with other headaches at T0 (2 primary stabbing headaches and 1 tension-type headache) that transformed to migraines at $\mathrm{T} 1$.

The proportion of patients with an attack duration over $4 \mathrm{~h}$ was consistently higher in T1, involving $75 \%$ versus $23 \%$ of the subject sample observed at T0. We found no significant differences in attack frequency between the two samples. Pain was more often unilateral at T1, involving $43.75 \%$ of subjects as compared to $17.6 \%$ of patients at $\mathrm{T} 0$, but the difference was not statistically significant. The quality of pain was mainly throbbing, and it was significantly different from that reported in the preschooler sample. We found no significant difference in pain intensity, pain increased by movement or pain sites (mainly frontal side) between T0 and T1. However, we observed that the pain site frequently shifted in the same subject from T0 to T1 (from frontal to temporal or vice versa, or from occipital to frontal or temporal sites in $75 \%$ of patients). As concerns features associated with migraine pain, no significant differences were found in the prevalence of vomit, nausea, phono and photophobia in migraineurs between $\mathrm{T} 0$ and $\mathrm{T} 1$. At $\mathrm{T} 1$, we also evaluated the prevalence of osmophobia (31.25\%), cranial autonomic symptoms (56.25\%), (the typical cranial autonomic signs that are a diagnostic criterion for TACs in ICHD3 ${ }^{20}$ ), and allodynia (68.7\%) referred to the site of pain during the painful attacks. Because these symptoms were not systematically evaluated in all patients at $\mathrm{TO}$, we could not compare the prevalence of these findings between the two study times. However, we tried to compare the prevalence of these features in the $\mathrm{T} 1$ patients and those in a new sample of migraineurs under age of 7 , taken from our recent study in which these features were evaluated [12] . The autonomic cranial symptoms were usually reported to accompany pain throughout its duration and were lateralized according to the site of pain (uni/bilateral) while the allodynia was referred to the area of pain.

We did not find any significant difference in the prevalence of osmophobia and cranial autonomic symptoms between these two groups [12]. However, the prevalence of allodynia was significantly increased in the sample at T1 $(68,7 \%)$ versus the sample of migraineurs under the age of 7 (20\%).

The family history for migraine was remarkable in $13 / 16$ (81.2\%) in the follow-up group. Almost all patients used symptomatic drugs to treat painful attacks, while only two used preventive treatments. 
Table 1

Distribution of migraineurs populations for age and gender.

\begin{tabular}{|c|c|c|c|c|c|}
\hline Migraineurs population & N. children & $\operatorname{sex}$ & Media age y. (ds) & range age & Follow-up media \\
\hline Migraineurs 2005 & 37 & $22 \mathrm{M} / 15 \mathrm{~F}$ & 4.8 & $1.5-5.11$ & \\
\hline interviewed Migraineurs 2005 at T0 & 17 & $7 \mathrm{M} / 10 \mathrm{~F}$ & 3.8 & $1.5-5.8$ & \\
\hline Migraineurs at $\mathrm{T} 1$ & 16 & $9 \mathrm{M} / 7 \mathrm{f}$ & 21.2 & $17-26$ & 17.4 \\
\hline
\end{tabular}

In Table 2, we summarize the main features of migraine sample at $\mathrm{T} 1$ as compared to first sample at T0 ${ }^{7}$ and to the new recent sample of migraineurs under the age of $7^{12}$.

\section{Discussion}

Follow-up studies of pediatric primary headaches in younger children (less than 6 years old) are rare, and are all based on shortterm evaluations $[8,9,16,17]$ ranging between 2 and 10 years, with an average of 3.5-7 years. The results of these studies showed generally that the early onset of migraine is usually an unfavorable prognostic factor, being frequently associated with the persistence of the symptoms at later ages. Early somatic symptoms, psychiatric comorbidity and vomiting were all found to predict the persistence of migraine. However, these studies present several limitations due to their small sample sizes [17], lack of use of IHS criteria [16], retrospective recall [20], short-term follow-up [8,9,16,17], selection population bias [20], and restricted age groups [9]. Our study is the first long-term follow-up carried out with migraine patients under the age of 6 years. Unfortunately, it does not interest the whole starting cohort due to the high rate of drop-out. and is comparable in follow-up duration to the study by Bille et al. [21]. Although reporting on a 4-fold larger sample size with no follow-up dropout, however, these authors enrolled patients who did not fulfill the IHS classification criteria, and, as compared to our cohort, explored children with higher age at the first observation (from 7 years on), and later migraine onset (6 years vs 3.8 years). Another study [22] was carried out on 1894 pediatric subjects (seven years or older) identified as migrainous patients according to Valhquist's criteria. This study was characterized by a $56.8 \%$ dropout rate, and presented further limitations due to its missing information about the onset age of migraine, and the somewhat lower accuracy of its data collection. Indeed, interviews were performed by non-specialist at all stages of the study, while basic information was acquired by selfreport questionnaire performed directly during school hours, or sent by mail.

During our follow-up study, we lost $51 / 74$ patients in the total original sample (69\%) and 20/37 (54\%) of the early migraine group.

Table 2

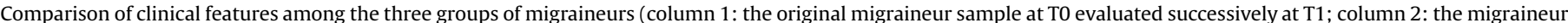
sample at T1 adding three subjects with changed headache in migraine $(13+3)$; column 3 : our other migraineur sample younger $7 \mathrm{y}$. successively published).

\begin{tabular}{|c|c|c|c|c|}
\hline Clinical features & $\begin{array}{l}\text { Original Migraineurs }<6 \text { years of age at } \mathrm{F} \\
\text { T0 } \\
17 \text { subjects (\%) } \\
\text { Column } 1 \\
\text { Raieli et al., } 2005 \text { (ref. 7) }\end{array}$ & $\begin{array}{l}\text { Follow-up Migraine } \\
\text { group } \\
16 \text { subjects }(\%) \\
\text { Column } 2\end{array}$ & $\begin{array}{l}\text { Migraineurs children ( }<7 \text { years of } \\
\text { age) } \\
40 \text { subjects (\%) } \\
\text { Column } 3 \\
\text { Raieli et al., } 2015 \text { (ref. } 12 \text { ) }\end{array}$ & $\begin{array}{l}\mathrm{P}<0.05 \\
\text { Comparison between } \mathrm{col} .1 / \mathrm{col} 2 \text { or } \operatorname{col} 2 / \\
\text { col.3 }\end{array}$ \\
\hline Males/Females & $9 / 8$ & $9 / 7$ & $20 / 20$ & \\
\hline \multicolumn{5}{|l|}{ Duration attacks } \\
\hline$<4 \mathrm{~h}$ & $13(77)$ & $4(25)$ & $33(82.5)$ & \multirow[t]{2}{*}{$\mathrm{P}<0.031$ (1 column/2colomn) } \\
\hline$>4 \mathrm{~h}$ & $4(23)$ & $12(75)$ & $7(17.5)$ & \\
\hline \multicolumn{5}{|c|}{ Frequency attacks for months } \\
\hline$<4$ attacks & $10(58.8)$ & $9(56.25)$ & $19(50.0)$ & \multirow{4}{*}{ NS } \\
\hline$>4$ attacks & $7(42.2)$ & $7(43.75)$ & $21(50.0)$ & \\
\hline Migraine familiarity & $15(88.2)$ & $14(87.5)$ & $39(97.5)$ & \\
\hline Aura & $2(11)$ & $3(18.7)$ & $2(5.0)$ & \\
\hline \multicolumn{5}{|l|}{ Pain localization } \\
\hline Frontal & $8(90)$ & $10(62.5)$ & & \\
\hline Temporal & $5(10)$ & $5(31.25)$ & & \\
\hline other & $4(23.5)$ & $1(6.25)$ & & \\
\hline Unilaterality pain & $3(17.6)$ & $7(43.75)$ & $9(22.5)$ & \\
\hline \multicolumn{5}{|l|}{ Quality of pain } \\
\hline Throbbing & $7(41)$ & $14(87.5)$ & $25 / 40(62.5)$ & \multirow[t]{3}{*}{$\mathrm{P}<0.0057$ (1 column/2column) } \\
\hline Gravative & $6(35.3)$ & $2(12.5)$ & $6 / 40(15)$ & \\
\hline Not described & $4(23.5)$ & - & $9 / 40(22.5)$ & \\
\hline Physical activity & $9(53)$ & $10(62.5)$ & $24(60)$ & NS \\
\hline \multicolumn{5}{|l|}{ Intensity pain } \\
\hline severe & $12(70)$ & $12(75)$ & $30(75.0)$ & \multirow[t]{2}{*}{ NS } \\
\hline moderate & $5(30)$ & $4(25)$ & $10(25)$ & \\
\hline Vomiting & $6(36)$ & $3(18.75)$ & $18(45,0)$ & NS \\
\hline Nausea & $4(24)$ & $7(43.75)$ & $21(52.5)$ & NS \\
\hline Phonophobia & $10(58.8)$ & $10(62.5)$ & $31(77.5)$ & NS \\
\hline Photophobia & $11(64.7)$ & $10(62.5)$ & $29(72.5)$ & NS \\
\hline Osmophobia & & $5(31.25)$ & $10(25)$ & NS \\
\hline Cranial allodynia & $\mathrm{N} / \mathrm{Q}$ & $11(68.75)$ & $8(20,0)$ & $\mathrm{P}<0.0011$ (2column/3column) \\
\hline $\begin{array}{l}\text { Cranial autonomic } \\
\text { symptoms }\end{array}$ & $\mathrm{N} / \mathrm{Q}$ & $9(56.25)$ & $22(55.0)$ & NS \\
\hline Comorbidities & $9(53)$ & $11(68.75)$ & & NS \\
\hline Symptomatic Drugs & $7(41.1)$ & $14(87.25)$ & & $\mathrm{P}<0.0057$ (1 column/2column) \\
\hline Preventive drugs & 0 & $2(12.5)$ & & NS \\
\hline
\end{tabular}

NS: not significative; N/Q: not quantified. 
This migraine dropout percentage was slightly higher than those reported by Battistella [8] (34\%) and Virtanen [9] (37.5\%) which had shorter mean follow-up periods of 3.5 years and 7 years, respectively.

Our study revealed the persistence of migraine in about $76.4 \%$ of patients, a rate consistently higher than that reported by Bille [21], who found $32 \%$ of patients with lasting migraine at follow-up [19]. Moreover, in our study, migraineurs reported continuous, quite stable disease without consistent pain-free intervals, which was distinct from the fluctuating course described by Bille [21]. We did not find sex differences in patients with persistent migraine in adulthood. Conversely, in a Finnish study [22], childhood migraine was found to persist in $65 \%$ of females and $21 \%$ of males in the original sample of children, who were followed from age 7 to age 32. Therefore, our results suggest that the early onset of migraine (under age of 6 years) represents per se an important unfavorable prognostic factor for migraine persistency in young adulthood, independent of sex.

However, as the number of migraineurs is very prevalent, it cannot be excluded that the unfavorable prognosis could be related to the early appearance of headache and not to a specific headache type, as reported by Balottin et al. [17].In his dataset, numerically similar to that of our study, the headache was found to persist more in subjects with tension headache and generally improved with time. In favor of our hypothesis, in addition to the much longer duration of follow-up, it is to consider that the few cases of other primary headaches recruited to follow-up, either moved to migraines or remitted. Surely, studies on a larger population and including the different types of primary headache are needed to better explore this point and clarify the real prognostic role of early preschoolers' migraine.

Our data are supported by the observation that in adulthood, a higher frequency of attacks is associated with early onset of migraine ( $<7$ years) [23]. In the short-term follow-up studies, the persistence of migraine ranged between $56 \%$ and $61 \%$ of patients. Hernandez-Latorre et al. [16] found a more unfavorable course in migraineurs less than 6 years of age after 2-10 years of follow-up (30\% of dropouts). However, the authors did not specify the meaning of "favorable course" (lack of attacks or significant reduction in frequency of attacks), and reported that $41.3 \%$ of an unspecified number of children followed for more than 5 years experienced an absence of painful attacks that lasted up to 1 year. Battistella et al. [8] also reported unfavorable evolution in $51 \%$ of patients (with stable attack frequency and a lack of pain-free periods).

Differently from these studies, we found a consistently higher rate of unfavorable outcomes, as migraine course improved in only $23 \%$ of subjects, while disease persisted in $76 \%$ of them, with $38 \%$ showing unchanged and $38 \%$ worsened clinical patterns. Such high rate of persistency, considering the risk linked to early onset, could be dependent upon the very early migraine onset in our child sample. Other considerations about outcome-predicting conditions concern gender and family history of migraine. Our population showed the same proportion of male and female sufferers, so the data suggest that after many years, the early onset of migraine is an unfavorable predictor in males as well. This likely emphasizes the relevance of genetic factors, especially in childhood migraines, and seems to fit well with the observation that, in agreement with a previous study [24], more than $90 \%$ of our cohort had a remarkable family history of migraine. This further supports the possible link between familiarity for migraine with early onset of headache, which in turn forecasts severe migraine attacks in adulthood [24].

Finally in the follow-up the migraineurs sample increased by three subjects suffering previously from other types of headache, now transformed into migraine. This is in agreement with the data by
Battistella et al. [8] that showed these possible changes and suggested a clinical continuum between one headache form and another with underlaying common pathophysiological mechanisms. This is also observed in the primary headaches of adults[25].

A careful search for possible changes in the main characteristics of migraine episodes revealed an increase in the duration of painful attacks. This finding was, however, reported in previous comparative studies of younger and older children $[8,9,12]$. We found that other clinical features of migraine attacks, mostly the localization of the headache, which may shift from the frontal to the temporal region or vice versa, may change. The quality of the pain may also more often become pulsating. Similar data were reported in Virtanen's study [9], who also found changes in other migraine features not evaluated in our study. Different results (except for vomiting, which was significantly different) were reported by Eiditz-Markus et al. [10]. However, in their investigation, the comparison was performed among three samples of different ages, while in our study the comparison was intra-group (before/after). In our follow-up, we found a remarkable prevalence of allodynia (about 70\%) and autonomic cranial disorders (56,25\%). Unfortunately, we could not perform a comparison with the prevalence rates of such symptoms in the earlier sample at time T0, because these clinical features were not systematically investigated by us at that time, neither in other studies [5-11,13,16,17]. However, to have some knowledge, even if indirect, of changes in these features across time, we compared the prevalence of allodynia and cranial autonomic symptoms observed at $\mathrm{T} 1$ with those observed in another population of migraineurs under 7 years, which we studied in a subsequent research project [12]. We found a noteworthy increase of allodynia in adulthood in our sample, whereas the prevalence of cranial autonomic symptoms remained unchanged. Our observations suggest that allodynia may be present in both younger and older children $[12,26,27]$ however, a longer duration of the disorder and the persistence of attacks likely increase the prevalence of allodynia through a further sensitization of pain-related structures [28].

The prevalence of cranial autonomic symptoms in our two groups is similar to that reported in other studies carried out in pediatric as well as in adult patients $[29,30]$ these findings suggest that such features can be specific traits in migraineur subgroups, independent of the course of disease. In light of these observations, allodynia may be interpreted as an unfavorable factor for migraine persistence and worsening, suggesting that its prevention can help improve the disease course. Alternately, the early emergence of cranial autonomic symptoms can be interpreted as a predictive factor of migraine persistence in successive follow-up, suggesting more intensive migraine treatment in order to avoid the worsening of the disorder and the appearance of further worsening factors such as allodynia.

Our study had some limitations due to its small sample size, selected clinical population and high dropout rate (>50\%). However, the sample size was similar to that of other studies $[6,10,17]$, and large samples have been explored only in a few studies and with less accurate methods, like self-administered questionnaires. The dropout rate was slightly higher than those reported in other studies, although the latter had shorter follow-up periods. The dropouts were mainly due to the fact that over the years many patients had changed the phone numbers registered in our archives).

Another limitation of the study may be due to the medical nature of the follow-up, based on retrospective medical records entrusted to the subjects, without documentation (such as diary papers, etc.) and the absence of intermediate interviews during this long-time interval between $\mathrm{T} 0$ and $\mathrm{T} 1$. Given the remitting nature of migraine it is possible that subjects currently suffering from 
migraine may have experienced more or less long periods of remission, of which they have no note. Considering that only 2 subjects had been given preventive therapy, according to the usually poor use of prophylaxis therapy in western countries, our data seem to describe the natural evolution of headache.

Of course, further studies are needed with larger population samples, with multicentric enrollment or population-based recruitment with long-term follow-up, and with intermediate control steps. Future investigations should also include the analysis of both genetic and environmental factors, such as twinning and fetal growth restriction, associated with migraine [31-32]. However, we are aware that achieving these goals is very difficult and requires a lot of effort.

\section{Conclusions}

To our knowledge, this is the first long-term follow-up study carried out on a population of migraineurs below the age of 6 years. The results suggest that the early onset of migraine is an unfavorable factor for its persistence in both male and female adults. Some clinical features may change during the clinical course, and the active persistence of the disorder may facilitate the potentiation of sensitizing neural-pain-related centers with increasing allodynia. The occurrence of cranial autonomic symptoms in preschool-age migraneurs may be a predictive factor of the persistence of the disorder. This finding suggests an intensive treatment for these children in order to avoid allodia and worsening of migraine.

In view of the high social impact of migraine, and the severe outcome of its early onset on quality of life, the topic appears interesting and, certainly worthy of being further explored in prospective studies with larger sample size, with the use of neurophysiologic markers, and with examination of more genetic and environmental variables.

\section{Author declaration}

We wish to confirm that there are no known conflicts of interest associated with this publication and there has been no significant financial support for this work that could have influenced its outcome.

\section{Declaration of conflicting interests}

The authors declared no potential conflicts of interest with respect to the research, authorship, and/or publication of this article.

\section{Funding}

The authors declared to have received no funder

\section{References}

[1] I. Abu-Arafeh, R. Howells, Primary headaches in children under the age of 7 years, Curr. Pain Headache Rep. 18 (2014) 401-408.

[2] M. Sillanpaa, P. Piekkala, P. Kero, Prevalence of headache at preschool age in an unselected child population, Cephalalgia 11 (1991) 239-242.

[3] I. Abu-Arefeh, G. Russell, Prevalence of headache and migraine in schoolchildren, BMJ 309 (1994) 765-769.

[4] M.A. Arruda, V. Guidetti, F. Galli, R.C. Albuquerque, M.E. Bigal, Primary headaches in childhood: a population-based study, Cephalalgia 30 (2010) $1056-1064$.
[5] M.L. Chu, S. Shinnar, Headaches in children younger than 7 years of age, Arch. Neurol. 49 (1992) 79-82.

[6] U. Balottin, F. Nicoli, G. Pitillo, O. Ferrari Ginevra, R. Borgatti, G. Lanzi, Migraine and tension headache in children under 6 years of age, Eur. J. Pain 8 (2004) 307-314.

[7] V. Raieli, M. Eliseo, E. Pandolfi, M. La Vecchia, G. La Franca, D. Puma, et al., Recurrent and chronic headaches in children below 6 years of age, J. Headache Pain 6 (2005), 135-4.

[8] P.A. Battistella, E. Fiumana, M. Binelli, E. Bertossi, P. Battista, E. Perakis, et al., Primary headaches in preschool age children: clinical study and follow-up in 163 patients, Cephalalgia 26 (2006) 162-171.

[9] R. Virtanen, M. Aromaa, P. Rautava, L. Metsähonkala, P. Anttila, H. Helenius, et al., Changing headache from preschool age to puberty. A controlled study, Cephalalgia 27 (2007) 294-303.

[10] T. Eidlitz-Markus, O. Gorali, Y. Haimi-Cohen, A. Zeharia, Symptoms of migraine in the paediatric population by age group, Cephalalgia 28 (2008) $1259-1263$.

[11] S. Ramdas, M. Prasad, I. Abu-Arafeh, Primary headache disorders in children under 7 years of age, Scot. Med. J. 58 (2013) 26-29.

[12] V. Raieli, R. Pitino, G. Giordano, C. Spitalieri, F. Consolo, D. Puma, G. Santangelo, F. Vanadia, M. D'Amelio, Migraine in a pediatric population: a clinical study in children younger than 7 years of age, Dev. Med. Child Neurol. 57 (2015) 585-588.

[13] R. Torriero, A. Capuano, R. Mariani, R. Frusciante, S. Tarantino, L. Papetti, F. Vigevano, M. Valeriani, Diagnosis of primary headache in children younger than 6years: a clinical challenge, Cephalalgia 37 (2017) 947-954.

[14] E. Correnti, F. Drago, L.M. Messina, L. Rocchitelli, F. Daiuto, F. Vanadia, V. Raieli, Headaches in preschoolers: are red flags predictive of positive neuroimaging in emergency department? Scifed J.Headache and Pain 1 (2018) 1-5.

[15] V. Raieli, A. Compagno, E. Pandolfi, M. La Vecchia, D. Puma, G. La Franca, D. Ragusa, Headache: what do children and mothers expect from pediatricians? Headache 50 (2010) 290-300.

[16] M.A. Hernandez-Latorre, M. Roig, Natural history of migraine in childhood, Cephalalgia 20 (2000) 573-579.

[17] U. Balottin, C. Termine, F. Vicoli, Idiopathic headache in children under six years of age: a follow-up study, Headache 45 (2005) 705-715.

[18] Raieli V, Eliseo GL, Pandolfi E, Puma D, Ragusa D and Eliseo M. The diagnostic flow-chart in headaches: use of a semi-structured interview in pediatric population. [Italian.]. RivNeurologia1998; 8:77-82.

[19] Headache classification committee of the international headache society (IHS) the international classification of headache disorders, 1st edition, Cephalalgia 8 (1988) 1-96.

[20] Headache classification committee of the international headache society (IHS) the international classification of headache disorders, 3rd edition (beta version), Cephalalgia 33 (2013), 629-808.

[21] B. Bille, A 40-year follow-up of school children with migraine, Cephalalgia 17 (1997) 488-491.

[22] M. Sillanpaa, M.M. Saarinen, Long term outcome of childhood onset headache: a prospective community study, Cephalalgia 38 (2018) 1159-1166.

[23] C. Asuni, M. Manchia, A. Deidda, M.E. Stochino, A. Cherchi, M. del Zompo, Mixture analysis of age at onset in migraine without aura: evidence for three subgroups, Headache 50 (2010) 1313-1319.

[24] T. Eidlitz-Markus, Y. Haimi-Cohen, A. Zeharia, Association of age at onset of migraine with family history of migraine in children attending a pediatric headache clinic: a retrospective cohort study, Cephalalgia 35 (2015) 722-727.

[25] A.L. Vollesen, S. Benemei, F. Cortese, A. Labastida-Ramírez, F. Marchese, L. Pellesi, M. Romoli, M. Ashina, C. Lampl, School of advanced studies of the European headache federation (EHF-SAS). Migraine and cluster headache the common link, J. Headache Pain 19 (1) (2018 Sep 21) 89, 0.1186/s10194018-0909-4.

[26] T. Eidlitz-Markus, A. Shuper, O. Gorali, A. Zeharia, Migraine and cephalic cutaneous allodynia in pediatric patients, Headache 47 (2007) 1219-1223.

[27] V. Raieli, D. Trapolino, G. Giordano, C. Spitaleri, F. Consolo, G. Santangelo, D. Buffa, F. Vanadia, M. D'Amelio, Juvenile migraine and allodynia: results of a retrospective study, Headache 55 (2015) 413-418.

[28] M.A. Louter, J.E. Bosker, W.P. van Oosterhout, E.W. van Zwet, F.G. Zitman, M.D. Ferrari, G.M. Terwindt, Cutaneous allodynia as a predictor of migraine chronification, Brain 136 (2013) 3489-3496.

[29] P. Barbanti, G. Fabbrini, M. Pesare, N. Vanacore, R. Cerbo, Unilateral cranial autonomic symptoms in migraine, Cephalalgia 22 (2002) 256-259.

[30] A.A. Gelfand, A.C. Reider, P.J. Goadsby, Cranial autonomic symptoms in pediatric migraine are the rule, not exception, Neurology 81 (2013) 1-6.

[31] G. Puccio, M. Giuffré, M. Piccione, E. Piro, V. Malerba, G. Corsello, Intrauterine growth pattern and birth weight discordance in twin pregnancies: a retrospective study, Ital. J. Pediatr. 40 (2014) 43.

[32] BørteS, B.S. Winsvold, S.Ø. Stensland, M.C. Småstuen, J.A. Zwart, The effect of foetal growth restriction in the development of migraine and tension-type headache in adulthood. The HUNT Study, PloS One 12 (2017) 1-12. 